\title{
SODA: 6TiSCH Open Data Action
}

\author{
Mališa Vučinić*, Milica Pejanović-Djurišić*, Thomas Watteyne ${ }^{\dagger}$ \\ * University of Montenegro, Podgorica, Montenegro \\ $\dagger$ EVA team, Inria, Paris, France \\ E-mail: malisav@ac.me,milica@ac.me, thomas.watteyne@inria.fr
}

\begin{abstract}
Industrial-grade performance of wireless networks has become a reality of the open Internet of Things through a low-power wireless technology called 6TiSCH. A standardization group of the same name within the Internet Engineering Task Force (IETF) has been working on a zero-configuration, IPv6enabled solution applicable to industrial monitoring and control, smart building, home, city and agriculture. The base standardization work is virtually done. Before different stakeholders invest significant resources in developing and deploying 6TiSCHbased solutions, they need to have a certain level of assurance about whether the technology indeed meets their requirements. An unbiased performance benchmark of a standards-compliant 6TiSCH solution is therefore required by the industry, the research community and the standardization group. 6TiSCH Open Data Action (SODA) is a research project that will provide a $6 \mathrm{TiSCH}$ benchmark and open datasets in different industryrelevant scenarios. SODA will automate the experimentation on these scenarios so benchmarking can remain up-to-date with changes to the standards, and facilitate comparison with future technological developments, resulting in continuous delivery benchmarking. This paper introduces the SODA project and seeks community involvement on the definition of scenarios and Key Performance Indicators, and on the usage of the tools that will be provided.
\end{abstract}

\section{INTRODUCTION}

Industrial Internet of Things (IIoT) solutions require reliable, low-power and secure wireless networking. Today, this is achieved using industrial standards such as WirelessHART and ISA100.11a, both of which use the Time-Slotted Channel Hopping (TSCH) mode of IEEE802.15.4. In TSCH networks, nodes form a mesh, they are tightly synchronized, time is sliced into timeslots, and a communication schedule tells each node what to do in a timeslot: transmit, listen or sleep. Transmission frequency is constantly changing, resulting in frequency diversity, a technique called "channel hopping".

Tens of thousands of TSCH-based networks are deployed today $^{1}$; they yield wire-like reliability and over a decade of battery lifetime [1]. The 6TiSCH standardization group within the Internet Engineering Task Force (IETF) ${ }^{2}$ couples this industrial-grade performance with the ease of use of IPv6.

The 6TiSCH working group has been standardizing a number of open Internet standards (RFCs) since its creation in 2013. These standards define the missing pieces towards

\footnotetext{
${ }^{1}$ One vendor alone, Emerson, reports over 36,300 networks deployed and over 10 billion operating hours: http://www.emerson.com/en-us/ expertise/automation/industrial-internet-things/pervasive-sensing-solutions/ wireless-technology.

2 https://datatracker.ietf.org/wg/6tisch/charter/
}

interoperable and autonomic operation of TSCH in IPv6 networks. Apart from IIoT, 6TiSCH also targets smart building, smart home, smart city and smart agriculture applications. 6TiSCH is fundamentally different from WirelessHART and ISA100.11a: communication schedule in 6TiSCH is built in a distributed manner. Each node in a 6TiSCH network, through the scheduling function it uses, decides locally what communication resources it needs. Because the networking paradigms they use are so different, the performance of $6 \mathrm{TiSCH}$ is not necessarily the same as the performance of WirelessHART and ISA100.11a.

Yet, companies want to know whether 6TiSCH meets their requirements. They ask questions such as Can 6TiSCH be used to build a long linear network along a road or a 200-meter long train? Does 6TiSCH offer low enough latency for home lightning or industrial control loops? How long does it take to build a 100-node network in a factory? Today, we do not have systematic answers to these questions.

At the same time, the academic community around lowpower wireless networking has launched an initiative to standardize how benchmarking is done, to minimize commonly biased results in academic papers [2]. Different research projects tackle the development and deployment of generic research tools that automate the experimentation ${ }^{3}$ and testing [3]. That is why the 6TiSCH Open Data Action (SODA) project aims at serving as a bridge between the academic initiative and existing tools and testbeds.

The goal of the SODA project is to lower the barrier for wide adoption of $6 \mathrm{TiSCH}$. SODA will provide a reference benchmark methodology and the datasets of standardscompliant 6TiSCH solutions, in industry-relevant test scenarios and conditions. SODA will automate the execution of these scenarios so benchmarking can remain up-to-date with changes to the standards, thereby facilitating comparison between older and newer proposals, resulting in "continuous delivery benchmarking". This paper gives an overview of the SODA project, and discusses the need for community involvement.

The remainder of this paper is organized as follows. Section II gives an overview of 6TiSCH, the state-of-the-practice and state-of-the-art. Section III introduces the SODA project and its expected outputs. Section IV discusses the community involvement that SODA is fostering. Finally, Section V concludes this paper.

\footnotetext{
3 www.fed4fire.eu
} 


\section{6TISCH: A PRIMER}

The 6TiSCH protocol stack is rooted in IEEE802.15.4 hardware that enables low-power wireless communication. The state-of-the-art IEEE802.15.4 radio transceivers consume less than $5 \mathrm{~mA}^{4,5}$ when transmitting or receiving. While low, this consumption allows a device to run off a pair of AA batteries for about a month, if the radio transceiver is always on. To extend this lifetime to a decade, the TSCH Medium Access Control (MAC) layer heavily duty-cycles the radio. The IEEE802.15.4 standard defines the operation of a node for the different timeslot types (transmit, listen, sleep) but it does not define how the schedule is built.

The 6TiSCH standardization group within the IETF fills this gap. Contrary to existing industrial solutions that use a central entity to calculate the network schedule, $6 \mathrm{TiSCH}$ relies on distributed scheduling. The $6 \mathrm{TiSCH}$ protocol stack is composed of 3 major components: 1) the 6top Protocol [4], allowing neighbor nodes to make changes to their schedule; 2) a scheduling function [5]-[8], an algorithm that drives the 6top Protocol; and 3) the secure join solution [9], allowing new nodes to securely join the network and obtain keys used for encryption.

6TiSCH is supported, or support is actively being worked on, by all major open-source Internet of Things projects [10]: OpenWSN, Contiki, Contiki-NG, RIOT, TinyOS. Together with ETSI, the 6TiSCH standardization group has so far organized 4 interoperability test events, in which different entities meet and test their 6TiSCH implementations for interoperability.

The research community around 6TiSCH keeps up with the standardization work. Novel proposals have recently been published that relate to scheduling functions [11]-[13], join procedures [14], [15], routing [16], and co-existence [17], to illustrate some. Different research projects also tackle aspects of 6TiSCH. For example, the H2020 F-Interop project ${ }^{6}$ devel- $^{-}$ ops tools for remote interoperability and conformance testing. F-Interop develops a "6TiSCH testing tool", which covers all aspects of the 6TiSCH stack. The H2020 ARMOUR project ${ }^{7}$ tackles security aspects of $6 \mathrm{TiSCH}$, particularly of its secure join solution.

\section{SODA OVERVIEW}

The SODA project has three main objectives:

1) Capture industry use cases and requirements, and map them to test scenarios and Key Performance Indicators (KPIs).

2) Collect and analyze performance data of a standardscompliant 6TiSCH stack in these scenarios, under different conditions.

3) Automate data collection and processing to enable "continuous delivery benchmarking".

\footnotetext{
4 www.linear.com/docs $/ 41870$

5 www.st.com/en/microcontrollers/stm32wb-series.html

${ }^{6}$ www.f-interop.eu

7 www.armour-project.eu
}

The challenge of Objective 1 is to define scenarios that are relevant for the industry, and at the same time enable future experimentation. We need KPIs that provide confidence to industry stakeholders for adopting 6TiSCH for their IoT products, yet facilitate future developments and comparisons. We discuss these aspects in detail in Sections III-B and III-C.

We define generic test scenarios to be able to provide a common ground to technology developers for future experimentation. We will provide a mapping of these scenarios to specific testbeds, as well as to the 6TiSCH simulator, a Python-based tool that answers what-if questions through quick performance estimation [18]. We will collect real-world data by executing these scenarios on different testbeds. Similarly, we will collect simulation data using different propagation models, including real-world traces [19] reproduced by the $6 \mathrm{TiSCH}$ simulator. This approach allows immediate comparison between realworld results and simulations, the isolation of the effects of propagation conditions and models on different KPIs, and experimentation in industry-relevant conditions. We discuss this in more details in Section III-D.

To meet Objective 3, we will complement the existing development projects and provide tools that will automate the benchmarking process on the testbeds and in the $6 \mathrm{TiSCH}$ simulator. We discuss these tools in Section III-E.

\section{A. Benchmark}

A SODA benchmark consists of 1) a test scenario, 2) a set of KPIs, 3) a test environment, and 4) a 6TiSCH implementation. In an ideal case, the latter would have little influence on the benchmark results, as one may expect the standards to determine the behavior of implementation(s). The practical factors such as implementation choices taken by different developers and stochastic propagation conditions necessarily add a level of indeterminism.

As part of the SODA project, we will focus on the OpenWSN stack [20], ETSI's reference implementation of 6TiSCH. We will put in place all the mechanisms for the community to complement our efforts by providing benchmarks using other available 6TiSCH implementations. We will facilitate this by fully automating the benchmarking process through different tools and a user interface (see III-E), exposing an API and using a standardized data format that a 6TiSCH implementation must implement in order to be integrated with our "continuous delivery benchmarking" tools. This will allow easy comparisons of different 6TiSCH implementations, and will highlight the effects of implementation choices and stochastic propagation conditions on performance.

\section{B. Test Scenarios}

The starting point for SODA is the definition of generic test scenarios. Under a "scenario", we want to emulate an industrial use case and typical operating conditions. We illustrate a preliminary list of parameters that each scenario encompasses in Table I. We aim at defining scenarios that correspond to typical "smart factory", "smart agriculture" and "smart city" deployments. For example, RFC5673 discusses 


\begin{tabular}{c|c} 
Parameter & Challenge \\
\hline \hline Number of nodes & Upper-bound set by a testbed \\
\hline Application traffic pattern and load & Within firmware \\
\hline Coverage requirement \\
(e.g. in number of hops) & $\begin{array}{c}\text { Testbed-dependent } \\
\text { transmit power }\end{array}$ \\
\hline Interference pattern and load & Testbed-dependent \\
\hline \hline
\end{tabular}

TABLE I

A TEST SCENARIO.

the requirements of the industrial low-power wireless networks that $6 \mathrm{TiSCH}$ targets. It states that $90 \%$ of the market is covered by packet rates between $1 /$ second and 1 /hour, with the average under $1 /$ minute. Similarly, we can derive typical coverage requirements (e.g. in maximum number of hops in the network) and interference levels.

Each of these scenarios is then "mapped" to a specific test environment (e.g. a testbed or the simulator). This corresponds to the precise definition of testbed nodes in use, the transmit radio power needed to meet the hop (i.e. coverage) requirement, and any other testbed-specific parameter needed to run the experiment.

\section{KPIs and Data Collection}

Industry-relevant KPIs for the evaluation of the wireless technology are typically end-to-end reliability, latency and radio duty cycle. As discussed by Duquennoy et al. [2], these are also commonly used by researchers for evaluating new proposals and comparisons with state-of-the-art. Another important KPI for a deployment is network formation time. Typically, this depends on the density of the deployment and the load of the bootstrap protocols.

With SODA, we will approach the data collection from a slightly different perspective than typically used by researchers. As we want to execute our scenarios on multiple testbeds, we aim at bypassing testbed-specific logging capabilities. Furthermore, we want our results to be easily comparable with real deployments where logging is not an option. For that reason, we plan on collecting all the data necessary to calculate high-level KPIs with over-the-air statistic reports, by having nodes in the network locally store and communicate in-band the necessary information. The nodes will periodically report to a central server, encoding the latest measurements. Obviously, with this approach, there is a risk that the reporting of a measurement (i.e. sending packets containing these reports) influences the system we are measuring (i.e. the network). We intend to study this further and find optimal values for the actual sampling period.

For example, to calculate latency, each application packet carries a timestamp of the instant when it was generated, which allows the server to calculate the delta. For reliability, the server needs to know the total number of application packets generated, which can be included in statistics reports, and compared to the actual number of received packets. Duty cycle in 6TiSCH networks is highly related to the communication schedule, so it is sufficient for a node to report how many cells it has installed with its neighbors. Network formation time can be computed by having the nodes report the timestamp of when they joined.

We will use the Concise Binary Object Representation (CBOR) format to encode these statistics in a flexible manner. Datasets which contain the statistics and application traffic will be published in an open-data approach.

\section{Test Environments}

As discussed in Section III, we will map the generic test scenarios to different test environments. We will map each scenario to 1) two testbeds with IEEE802.15.4-compliant devices, and 2) the 6TiSCH simulator. In the following, we give a brief overview of each of these test environments.

1) IoT-lab Testbed: Judging by the number of published works, IoT-lab ${ }^{8}$ in France has so far proven to be the defacto testbed for $6 \mathrm{TiSCH}$ experimentation. Many researchers already have custom automation scripts and tools developed to run their experiments on IoT-lab and process results. For this reason, we believe that for SODA to be a success, it must provide reference results on IoT-lab. We have identified that the Saclay deployment site of IoT-lab provides the most realistic propagation conditions and is the one where OpenWSN implementation has been most thoroughly tested on. SODA will use the IoT-lab Saclay site and its A8 Open Nodes that integrate an M3 Open Node with STM32F microcontroller and Atmel's AT86RF231 radio. We are also aware, however, of a drawback of IoT-lab. M3 Open Nodes provided by IoTlab have a technical limitation that prevents $6 \mathrm{TiSCH}$ with default timings to be executed with link-layer security enabled. Namely, hardware acceleration module for encryption on M3 Open Nodes is placed within the radio chip and accessed through SPI, introducing large delays. Encryption of a 127byte frame with hardware acceleration takes approximately $5 \mathrm{~ms}$, making it infeasible to run the default $10 \mathrm{~ms} \mathrm{TSCH}$ slots with security enabled.

2) w-iLab.t Testbed: We will also use the w-iLab. $t^{9}$ facility in Ghent, Belgium (see Fig. 1) to 1) study the effect of different propagation conditions on performance, and 2) overcome the discussed technical limitations of IoT-lab. More precisely, we will use the IoT-officelab deployment of w-iLab.t providing 120 nodes installed over three floors in a building. w-iLab.t provides 802.15.4-compliant Zolertia Re-Mote nodes, based on CC2538 System on Chip (SoC). This SoC supports $6 \mathrm{TiSCH}$ with default timings and link-layer security enabled. To illustrate, hardware-accelerated encryption of a 127-byte frame on CC2538 at $32 \mathrm{MHz}$ takes less than $60 \mu \mathrm{s}$.

3) The 6TiSCH Simulator: While testbeds provide results close to real deployments, they may not be the best suited tool for quickly testing an idea, due to the inherent overhead of firmware development and remote debugging. The $6 \mathrm{TiSCH}$ simulator ${ }^{10}$ was developed for precisely this purpose: to allow a quick performance estimation for a given configuration and a set of conditions [18]. We are currently extending

\footnotetext{
${ }^{8}$ https://www.iot-lab.info

${ }^{9}$ http://doc.ilabt.iminds.be/ilabt-documentation/wilabfacility.html

${ }^{10} \mathrm{https}: / /$ bitbucket.org/6tisch/simulator
} 


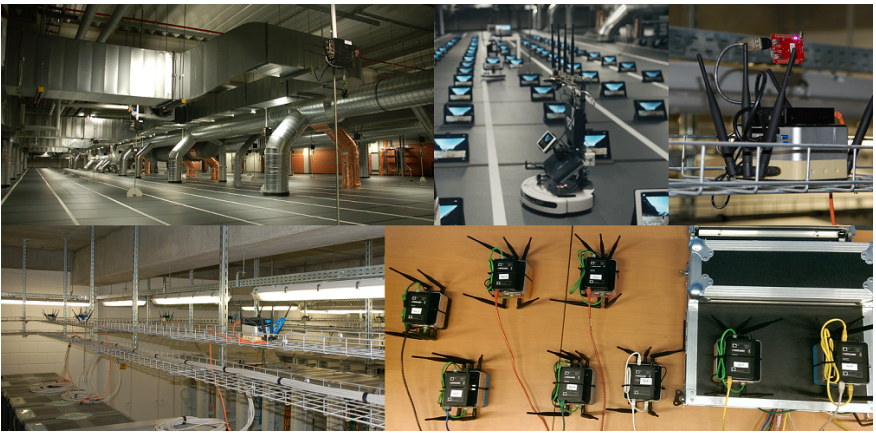

Fig. 1. An illustration of w-iLab.t facility (reproduced from the w-iLab.t website).

this simulator to support reproduction of connectivity traces obtained from real deployments [19]. This will allow us to run a given scenario on the exact same conditions as present in a real-world deployment or a testbed, and look for unexpected performance anomalies.

\section{E. Experimentation Tools}

Apart from the scientific component on studying network performance in different conditions and looking for anomalies, the SODA project will also provide different tools that will enable "continuous delivery benchmarking" on defined scenarios and test environments.

As discussed by Duquennoy et al. [2], a user should ideally select a scenario, upload/select a firmware image, and click "play". SODA will achieve this level of automation through the $\mathrm{jFed}^{11}$ tool provided by the Fed4Fire+ project that allows a federated access and experiment automation. We will also develop a web-based solution enabling a scenario to be executed on each of the considered testbeds, and the 6TiSCH simulator. We will automate the data collection and processing, and automate the graph generation through tools such as Grafana.

On the firmware side, SODA will develop an open-source statistics collection module for OpenWSN, the reference 6 TiSCH implementation, that will enable data collection on a generic testbed, as well as in real deployments.

\section{F. Users}

SODA takes a challenging approach by targeting the industry, the standardization bodies and the academic community.

On the one hand, industry stakeholders are typically interested in the performance of standards-compliant solutions; so are the standardization bodies that produce the actual specifications. For this reason, SODA will provide a benchmark of the OpenWSN implementation in different settings covering the base 6TiSCH specs without any additional optimizations. This output will serve the industry to asses whether $6 \mathrm{TiSCH}$ fits their requirements, as well as the standardization bodies on whether the produced specifications meet the expectations.

\footnotetext{
${ }^{11}$ https://www.fed4fire.eu/tools/jfed/
}

On the other hand, the research community is typically interested in the optimizations that result in performance improvements. The benchmarks provided by SODA will therefore serve to the community to assess the impact of these optimizations.

Through the tools SODA will develop, we will enable the "continuous delivery benchmarking", allowing the research community and industry stakeholders to quickly assess the impact of specific optimizations. Our tools will allow the user to immediately share the results or keep them proprietary. All of this comes with an added benefit of using a standardized benchmarking methodology, something that has been missing in our research community [2].

\section{Community Involvement}

Our goal is for the outputs of SODA to be used by the research community; we are looking for feedback on several topics.

First, the main requirement for scenario definitions is that they are relevant to the industry. Are we missing some important parameter to describe a scenario? Capturing multiple potential use cases and typical deployment requirements within a single parameter set is a tempting task. As discussed in Section III-B, the preliminary idea is to survey the existing smart factory, smart agriculture and smart city deployments, and to generalize each with a single parameter set. Can the same goal be achieved with a different approach?

Second, we are looking for KPIs that are interesting for future research. What information other than that discussed in Section III-C should we collect within the firmware statistics module in order to calculate research-relevant KPIs?

Third, we will automate the experimentation on two testbeds (IoT-lab and w-iLab.t) and on the 6TiSCH simulator, also providing an easy-to-use web interface for launching experiments and processing results. The community could develop support for additional IEEE802.15.4 testbeds and/or 6TiSCH implementations, and integrate it with the interface.

Lastly, we are looking for input on the debugging process and the evolution of the tools with future technological developments. How to best capture the firmware development and corresponding network debugging within a generic graphical interface? How to ensure that the benchmark and the tools are up-to-date with the state of 6TiSCH standards and trending research topics?

\section{CONClusion}

6TiSCH Open Data Action (SODA) will provide a reference benchmark and open datasets of a standards-compliant 6TiSCH solution, in different conditions and test environments. SODA will enable "continuous delivery benchmarking" through experiment and data processing automation. We are looking for community involvement both on making the results and tools relevant for the industry and the academic community, and on extending the support of our tools to additional test environments and 6TiSCH implementations. 


\section{REFERENCES}

[1] T. Watteyne, J. Weiss, L. Doherty, and J. Simon, "Industrial IEEE802. 15.4 e networks: Performance and trade-offs," in Communications (ICC), 2015 IEEE International Conference on. IEEE, 2015, pp. 604-609.

[2] S. Duquennoy, O. Landsiedel, C. A. Boano, M. Zimmerling, J. Beutel, M. C. Chan, O. Gnawali, M. Mohammad, L. Mottola, L. Thiele et al., "A benchmark for low-power wireless networking," in Proceedings of the 14th ACM Conference on Embedded Network Sensor Systems CDROM. ACM, 2016, pp. 332-333.

[3] S. Ziegler, S. Fdida, T. Watteyne, and C. Viho, "F-Interop-online conformance, interoperability and performance tests for the IoT," in Conference on Interoperability in IoT (InterIoT), 2016.

[4] Q. Wang, X. Vilajosana, and T. Watteyne, 6top Protocol (6P), Internet Engineering Task Force (IETF) Internet-Draft draft-ietf6tisch-6top-protocol-09, 2017, Work in Progress. [Online]. Available: https://datatracker.ietf.org/doc/html/draft-ietf-6tisch-6top-protocol-09

[5] T. Chang, M. Vučinić, and X. Vilajosana, 6TiSCH Minimal Scheduling Function (MSF), Internet Engineering Task Force (IETF) Internet-Draft draft-chang-6tisch-msf-00, 2017, Work in Progress. [Online]. Available: https://datatracker.ietf.org/doc/html/draft-chang-6tisch-msf-00

[6] D. Dujovne, L. A. Grieco, M. R. Palattella, and N. Accettura, 6TiSCH 6top Scheduling Function Zero / Experimental (SFX), Internet Engineering Task Force (IETF) Internet-Draft draft-ietf6tisch-6top-sfx-00, 2017, Work in Progress. [Online]. Available: https://datatracker.ietf.org/doc/html/draft-ietf-6tisch-6top-sfx-00

[7] S. Duquennoy, X. Vilajosana, and T. Watteyne, 6TiSCH Autonomous Scheduling Function (ASF), Internet Engineering Task Force Internet-Draft draft-duquennoy-6tisch-asf-01, 2018, work in Progress. [Online]. Available: https://datatracker.ietf.org/doc/html/ draft-duquennoy-6tisch-asf-01

[8] S. Anamalamudi, B. R. Liu, M. Zhang, A. R. Sangi, C. E. Perkins, and S. Anand, Scheduling Function One (SF1): hopby-hop Scheduling with RSVP-TE in 6tisch Networks, Internet Engineering Task Force (IETF) Internet-Draft draft-satish-6tisch6top-sf1-04, 2017, Work in Progress. [Online]. Available: https: //datatracker.ietf.org/doc/html/draft-satish-6tisch-6top-sf1-04

[9] M. Vučinić, J. Simon, K. Pister, and M. Richardson, Minimal Security Framework for 6TiSCH, Internet Engineering Task Force (IETF) Internet-Draft draft-ietf-6tisch-minimal-security-04, 2017, Work in Progress. [Online]. Available: https://datatracker.ietf.org/doc/html/ draft-ietf-6tisch-minimal-security-04

[10] T. Watteyne, V. Handziski, X. Vilajosana, S. Duquennoy, O. Hahm, E. Baccelli, and A. Wolisz, "Industrial wireless IP-based cyber-physical systems," Proceedings of the IEEE, vol. 104, no. 5, pp. 1025-1038, 2016.

[11] T. P. Duy, T. Dinh, and Y. Kim, "Distributed cell selection for scheduling function in 6tisch networks," Computer Standards \& Interfaces, vol. 53, pp. 80-88, 2017.

[12] G. Daneels, B. Spinnewyn, S. Latré, and J. Famaey, "ReSF: Recurrent Low-Latency Scheduling in IEEE 802.15. 4e TSCH networks," Ad Hoc Networks, vol. 69, pp. 100-114, 2018.

[13] K. Muraoka, T. Watteyne, N. Accettura, X. Vilajosana, and K. Pister, "Simple distributed scheduling with collision detection in TSCH networks," IEEE Sensors Journal, vol. 16, no. 15, pp. 5848-5849, 2016.

[14] B. Al Nahas, S. Duquennoy, and O. Landsiedel, "Network Bootstrapping and Leader Election in Low-power Wireless Networks," in ACM SenSys. ACM, 2017.

[15] M. Vučinić, T. Watteyne, and X. Vilajosana, "Broadcasting strategies in 6TiSCH networks," Internet Technology Letters, vol. 1, no. 1, 2018.

[16] F. Righetti, C. Vallati, G. Anastasi, and S. Das, "Performance Evaluation the 6top Protocol and Analysis of its Interplay with Routing," in Smart Computing (SMARTCOMP), 2017 IEEE International Conference on. IEEE, 2017, pp. 1-6.

[17] S. B. Yaala, F. Théoleyre, and R. Bouallegue, "Cooperative resynchronization to improve the reliability of colocated IEEE 802.15. 4-TSCH networks in dense deployments," Ad Hoc Networks, vol. 64, pp. 112126, 2017.

[18] E. Municio, G. Daneels, M. Vučinić, S. Latr, J. Famaey, K. Muraoka, X. Vilajosana, and T. Watteyne, "Simulating 6TiSCH Networks," To appear, 2018.

[19] K. Brun-Laguna, P. Henrique Gomes, P. Minet, and T. Watteyne, "Moving Beyond Testbeds? Lessons (We) Learned about Connectivity," To appear, 2018.
[20] T. Watteyne, X. Vilajosana, B. Kerkez, F. Chraim, K. Weekly, Q. Wang, S. Glaser, and K. Pister, "OpenWSN: a standards-based low-power wireless development environment," Transactions on Emerging Telecommunications Technologies, vol. 23, no. 5, pp. 480-493, 2012. 